\title{
Ethyl pyruvate protects against sepsis by regulating energy metabolism
}

\author{
This article was published in the following Dove Press journal: \\ Therapeutics and Clinical Risk Management \\ 23 February 2016 \\ Number of times this article has been viewed
}

\author{
Hongjun Kang' \\ Zhi Mao' \\ Yan Zhao' \\ Ting Yin ${ }^{2}$ \\ Qing Song' \\ Liang Pan' \\ Xin $\mathrm{Hu}^{\prime}$ \\ Jie $\mathrm{Hu}^{\prime}$ \\ Feihu Zhou' \\ 'Department of Critical Care \\ Medicine, Chinese PLA General \\ Hospital, Beijing, 'Department of \\ Critical Care Medicine, The Centre \\ Hospital of BaoTou, BaoTou, People's \\ Republic of China
}

\begin{abstract}
Background: Ethyl pyruvate (EP) is a derivative of pyruvic acid that has been demonstrated to be a potential scavenger of reactive oxygen species as well as an anti-inflammatory agent. In this study, we investigated the protective effects of EP and its role in regulating the energy metabolism in the livers of cecal-ligation-and-puncture-induced septic mice.
\end{abstract}

Methods: The animals were treated intraperitoneally with $0.2 \mathrm{~mL}$ of Ringer's lactate solution or an equivalent volume of Ringer's lactate solution containing EP immediately after cecal ligation and puncture. Each mouse in the Sham group was only subjected to a laparotomy. At 30-, 60-, 180-, and 360-minute time points, we measured the histopathological alterations of the intestines, and the plasma levels of interleukin (IL)-1 $\beta$, IL-6, IL-10, and tumor necrosis factor- $\alpha$, and the total antioxidative capacity, malondialdehyde content, and lactate and lactate/ pyruvate levels in livers. Furthermore, we detected the levels of adenosine triphosphate, total adenylate, and energy charge in the livers.

Results: Our results demonstrated that the administration of EP significantly improved the survival rate and reduced intestinal histological alterations. EP inhibited the plasma levels of IL-1 $\beta$, IL-6, and tumor necrosis factor- $\alpha$ and increased the IL-10 level. EP significantly inhibited the elevation of the malondialdehyde, lactate, and lactate/pyruvate levels and enhanced the total antioxidative capacity levels in the liver tissues. The downregulation of the adenosine triphosphate, total adenylate, and energy charge levels in the liver tissues was reversed in the septic mice treated with EP.

Conclusion: The results suggest that EP administration effectively modulates the energy metabolism, which may be an important component in treatment of sepsis.

Keywords: sepsis, ethyl pyruvate, energy metabolism, inflammation, reactive oxygen species

\section{Introduction}

Sepsis is an inflammatory and reactive oxygen species (ROS) reaction to a severe, life-threatening infection, and it induces organ dysfunction. ${ }^{1,2}$ Although there has been remarkable progress in the understanding of its pathophysiology, sepsis continues to pose serious clinical challenges and is the leading cause of death in intensive care units. The mechanisms of sepsis have been poorly understood, and no effective treatment has been developed.

Pyruvic acid $\left(\mathrm{CH}_{3} \mathrm{COCOOH}\right)$, a three-carbon alpha-keto monocarboxylic acid that is the final product of glycolysis, is present in cells and extracellular fluids as pyruvate. Pyruvate is not stable in an aqueous solvent and spontaneously forms a variety of products, some of which might be toxic. ${ }^{3}$ Ethyl pyruvate (EP) is a derivative of pyruvate, and in a Ringer's balanced calcium- and potassium-containing salt solution, it is stable and nontoxic. Previous studies demonstrated that a Ringer's balanced EP solution prevents structural and functional damage to the intestinal mucosa induced by
Correspondence: Feihu Zhou Department of Critical Care Medicine, Chinese PLA General Hospital, 28 Fuxing Road, Haidian District, Beijing 100853 , People's Republic of China

Tel +86 I38 I09| 8398

Fax +861068168834

Email feihuzhou30I@I26.com
Therapeutics and Clinical Risk Management 2016:12 287-294

Dovepress

http://dx.doi.org/10.2147/TCRM.S97989 (c) (1) (5) 2016 Kang et al. This work is published and licensed by Dove Medical Press Limited. The full terms of this license are available at https://www.dovepress.com/terms.php (c) hereby accept the Terms. Non-commercial uses of the work are permitted without any further permission from Dove Medical Press Limited, provided the work is properly attributed. For permission for commercial use of this work, please see paragraphs 4.2 and 5 of our Terms (https://www.dovepress.com/terms.php). 
mesenteric ischemia/reperfusion injury, ameliorates hepatic and intestinal mucosal lipid peroxidation, and prolongs the survival of rats. ${ }^{4,5}$ Additionally, EP treatment has been shown to increase the myocardial adenosine triphosphate (ATP) levels, reduce oxidative injury, diminish infarct size, and improve myocardial functions. ${ }^{6,7}$ These results demonstrated that EP prevents inflammation and free radical-mediated "cataract" formation both in vitro and in vivo. Thus, EP exhibits significant anti-inflammatory actions and is a potential scavenger of ROS.

Extensive evidence suggests that alterations in the uptake, utilization, and metabolism of glucose occur in various tissues during sepsis. ${ }^{8-10}$ Glucose is a fundamental element for the survival of all cells and tissues, and some intermediate products of the metabolism of glucose may be of benefit in severe sepsis and acute septic shock. Pyruvic acid is the final product of glycolysis and the starting substrate for the tricarboxylic acid cycle. Thus, EP is an inflammatory and oxygen radical regulator as well as an energy metabolism effector. The aim of this study was to elucidate the protective effects of EP against sepsis, particularly through regulation of the energy metabolism.

\section{Materials and methods}

\section{Animals}

Male Kunming mice (age, $40 \pm 1.5$ days; weight, $26 \pm 1.2 \mathrm{~g}$ ) were supplied by the Experimental Animal Center of the General Hospital of PLA. In our experiments, we followed the NIH Guide for the Care and Use of Laboratory Animals, which was approved by the Animal Care and Use Committee. All of the mice were housed in cages at $24^{\circ} \mathrm{C}-26^{\circ} \mathrm{C}$ with a constant day/ night rhythm and were given food and water ad libitum.

\section{Reagents}

The EP used in the experiments was purchased from Sigma-Aldrich (St Louis, MO, USA), and the colorimetric kits for malondialdehyde (MDA), the total antioxidative capacity (TAC), lactate, and pyruvate were purchased from the Jiancheng Bioengineering Institute (Nanjing, People's Republic of China). The radioimmunoassay kits for interleukin (IL)-1 $\beta$, IL-6, IL-10, and tumor necrosis factor- $\alpha$ (TNF- $\alpha$ ) were purchased from the Beifang Bioengineering Institute (Beijing, People's Republic of China). Other reagents were purchased locally and were of pure analytical grade.

\section{Murine model of sepsis}

To induce polymicrobial sepsis, we used an established murine model of cecal ligation and puncture (CLP), as previously described. ${ }^{11}$ Briefly, the mice were anesthetized with sodium pentobarbital (100 $\mathrm{mg} / \mathrm{kg}$, intraperitoneal). The peritoneum was opened, and the bowel was exposed. Two-thirds of the cecum was tied off and punctured once with a 19-gauge needle. Gentle pressure was applied at the perforation sites to extrude a small amount of feces, which was then returned to the peritoneal cavity. The laparotomy site was then stitched. Sham-operated mice underwent the same procedure, which included opening the peritoneum and exposing the bowel but did not include ligation and needle perforation of the cecum. The mice were randomly divided into the following three groups: the Sham group (Sham), the Sepsis group, in which the animals received $0.2 \mathrm{~mL}$ of Ringer's lactate solution (RL), and the Sepsis-and-EP treatment group, in which the animals received $0.2 \mathrm{~mL}$ of RL containing EP (75 mg/kg) (REPL) according to previous study. ${ }^{12}$ Intraperitoneal RL or REPL was administered immediately after the CLP procedures. The samples were collected immediately after Sham operation, and the RL and REPL groups were set for 30, 60, 180, and 360 minutes ( $n=20$ in each group). After the treatment, the time of death of each mouse was recorded for statistical mortality analysis if it died during its respective time point. At the end of time points, all of the mice that had survived were killed, and the samples were rapidly collected for analysis.

\section{Intestinal histopathological examination}

At different time points, the same intestinal segment $(4 \mathrm{~cm})$ from each mouse ( $\mathrm{n}=5$ for each group) was obtained and immediately placed in a $10 \%$ litmus-less formalin solution, and the same samples of intestinal tissue were embedded in paraffin blocks. The blocks were cut into $5 \mu \mathrm{m}$ thick sections and stained with hematoxylin and eosin. These stained sections were reviewed through light microscopy. The degree of injury to the intestinal tissues was evaluated, and each was graded from 0 to 5 , as described by Chiu et al. ${ }^{13}$

\section{Determination of the IL-I $\beta$, IL-6, IL-I0, and TNF- $\alpha$ levels in the plasma}

Whole blood was collected by extracting eyeballs from each mouse at different time points. After sitting for 30 minutes at room temperature, the whole blood was centrifuged at $3,000 \times g$ for 10 minutes at $4^{\circ} \mathrm{C}$, and the supernatants were collected and stored at $-20^{\circ} \mathrm{C}$. The concentrations of IL- $1 \beta$, IL-6, IL-10, and TNF- $\alpha$ in the plasma were determined using radioimmunoassay kits, according to the instructions provided by the manufacturer, and calculated with standard curves. 
Determination of the MDA, TAC, lactate, and pyruvate levels in liver homogenate

A total of $100 \mathrm{mg}$ of frozen liver tissues was immediately homogenized three times at $3913 \times g$ for 30 seconds in ten volumes of physiological saline using a high-speed homogenizer in an ice bath. The homogenates were centrifuged at $6,000 \times g$ for 10 minutes at $4^{\circ} \mathrm{C}$. The levels of MDA, TAC, lactate, and pyruvate in the supernatant were detected using their respective colorimetric assay kits, according to the manufacturer's recommendations.

\section{Determination of ATP,TAN, and EC in the liver}

The ATP, adenosine monophosphate (AMP), and adenosine diphosphate (ADP) levels in the liver samples were determined by high-performance liquid chromatography (HPLC), as previously described. ${ }^{14-16}$ In brief, $100 \mathrm{mg}$ of liver tissues frozen in liquid nitrogen was transferred into $1 \mathrm{~mL}$ of ice-cold $1 \mathrm{M}$ perchloric acid and was immediately homogenized using a homogenizer in an ice bath. After centrifugation $\left(15\right.$ minutes, $\left.4^{\circ} \mathrm{C}, 10,000 \times g\right)$, the supernatant was obtained, adjusted to $\mathrm{pH} 7$ with $6 \mathrm{M} \mathrm{NaOH}$, and vortexed for 20 seconds. The centrifugation process was repeated, and $50 \mu \mathrm{L}$ of supernatant was injected into the HPLC system. The mobile phase consisted of $50 \mathrm{mmol} / \mathrm{L} \mathrm{KH}_{2} \mathrm{PO}_{4}$, methanol, and an ion pair agent $\mathrm{A}(\mathrm{v}: \mathrm{v}: \mathrm{v}=74: 24: 2)$, a flow rate of $1 \mathrm{~mL} / \mathrm{min}$ was used, and the temperature of the chromatographic column was set to $25^{\circ} \mathrm{C}$. The HPLC chromatograms were obtained at a wavelength of $259 \mathrm{~nm}$. The concentrations were calculated by comparing the peak area of the sample peaks with the calibration curves for the peak areas of each standard compound. The total adenylate (TAN) and energy charge $(\mathrm{EC})$ levels were calculated as $\mathrm{TAN}=\mathrm{ATP}+\mathrm{AMP}+$ $\mathrm{ADP}$ and $\mathrm{EC}=(\mathrm{ATP}+1 / 2 \mathrm{ADP}) / \mathrm{TAN}$.

\section{Statistical analysis}

The Stata 7.0 software was used to process our data. The data were expressed as the mean \pm standard deviation of the results obtained from replicate treatments. The differences between datasets were assessed by a one-way analysis of variance. A log-rank test was used to analyze the survival. Differences with $P<0.05$ were considered statistically significant.

\section{Results}

\section{EP ameliorates CLP-induced intestine damage and survival rate}

To determine the protective effects of EP against CLPinduced sepsis, we detected the histopathological alterations in the intestines. As shown in Figure 1A and B, CLP induced time-dependent damage to the intestinal mucosa in mice. We observed massive inflammatory cell infiltration, epithelial necrosis, and lifting down of the sides of the villi in the RL treatment group compared with that of the Sham group. Although REPL treatment could not reverse the damage to the intestinal mucosa, it could improve the serious injury, particularly in the advanced stages. The survival rate of septic mice was significantly increased in the REPL treatment group compared to that of the RL treatment group (Figure 1C).

\section{EP attenuates the systemic inflammatory response to sepsis}

To identify the potential anti-inflammatory effects of EP, the plasma of each mouse was investigated. As shown in Figure 2, the levels of IL-1 $\beta$ (Figure 2A) and TNF (Figure 2B) significantly increased from 30 minutes and lasted until 360 minutes; the IL-6 (Figure 2C) levels were significantly increased starting from 180 minutes, and the IL-10 levels were significantly decreased starting from 60 minutes in the RL groups after CLP compared with the Sham group. The EP treatment significantly inhibited the IL-1 $\beta$, TNF, and IL- 6 levels but increased the IL-10 levels in the plasma of septic mice. Thus, EP can inhibit the pro-inflammatory factors that were released into the plasma and alleviated the systemic inflammatory reaction.

\section{EP reduces oxidative damage and metabolic acidosis in the liver of septic mice}

Of the effects of EP, its antioxidative effect has the most potential. We detected the TAC and MDA levels in liver homogenates. As shown in Figure 3, compared with those in the Sham group, the TAC (Figure 3A) levels were significantly decreased in the RL groups, and all of the MDA (Figure 3B) levels in the RL groups were significantly increased from 30 minutes after CLP to 360 minutes. The EP treatment significantly increased the TAC levels and inhibited the MDA levels in the livers of septic mice compared with the RL groups. We detected the levels of metabolic acidosis-associated lactate and lactate/pyruvate in the livers of the septic mice. As shown in Figure 3C, the levels of lactate and lactate/pyruvate in the livers of the RL group were significantly increased compared with those of the Sham group. The EP treatment reversed the metabolic acidosis, as indicated by the significantly reduced lactate and lactate/ pyruvate levels obtained after EP treatment compared with those of the RL groups. 

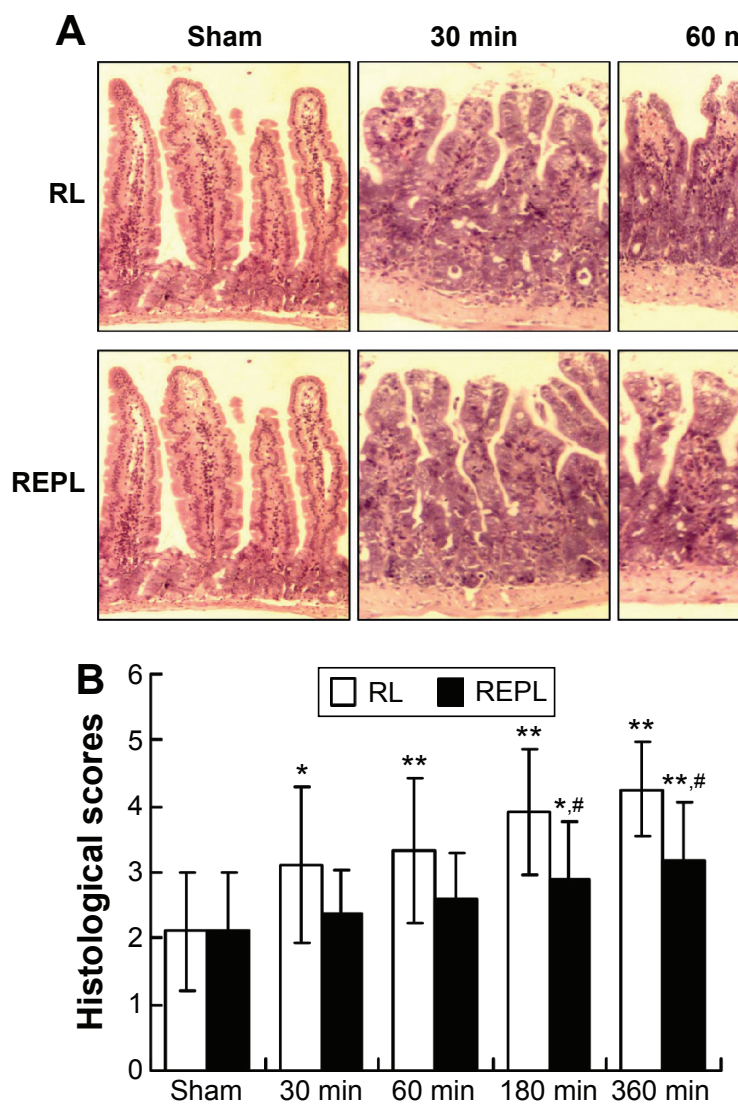
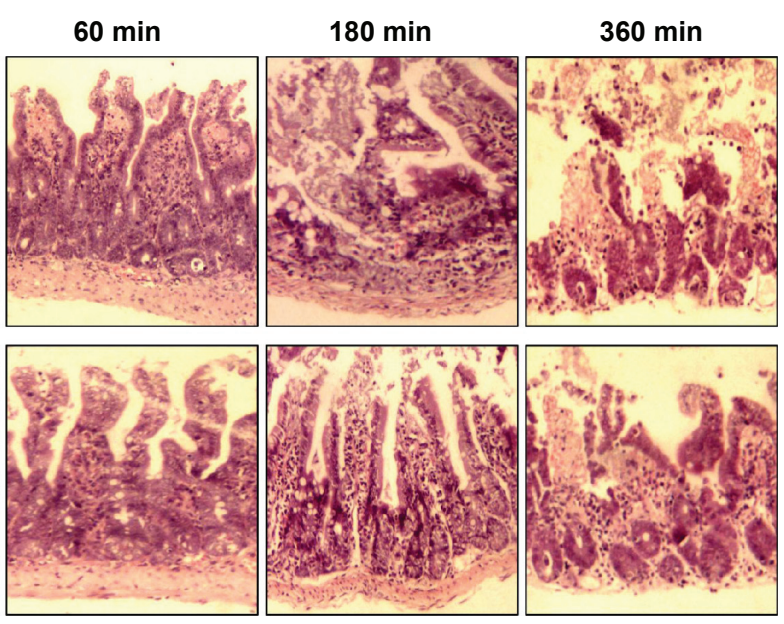

C

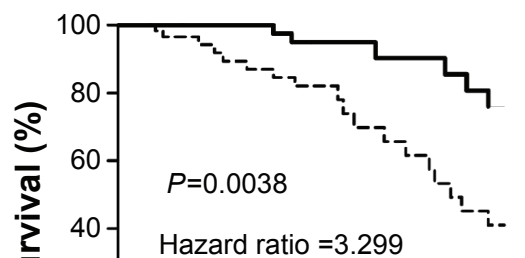

Hazard ratio $=3.299$

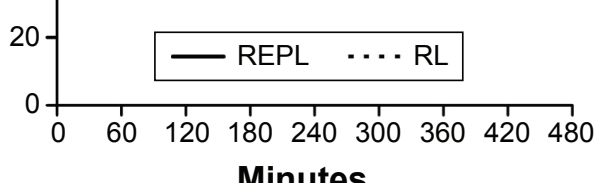

Minutes

Figure I Intestinal histopathological properties and survival rate.

Notes: (A) The intestinal sections were observed through hematoxylin and eosin staining. Representative physiological properties are shown. The original magnification was $\times 200$. (B) The normalized histopathological properties were analyzed as described by Chiu et al, ${ }^{13}$ and the results were present as the mean \pm SD ( $=5$ ). (C) The survival rate of each group ( $n=20$ for each group). ${ }^{*} P<0.05$ and $* * P<0.01$ versus the Sham group; ${ }^{*} P<0.05$.

Abbreviations: SD, standard deviation; RL, Ringer's lactate solution; REPL, RL containing ethyl pyruvate; min, minutes.
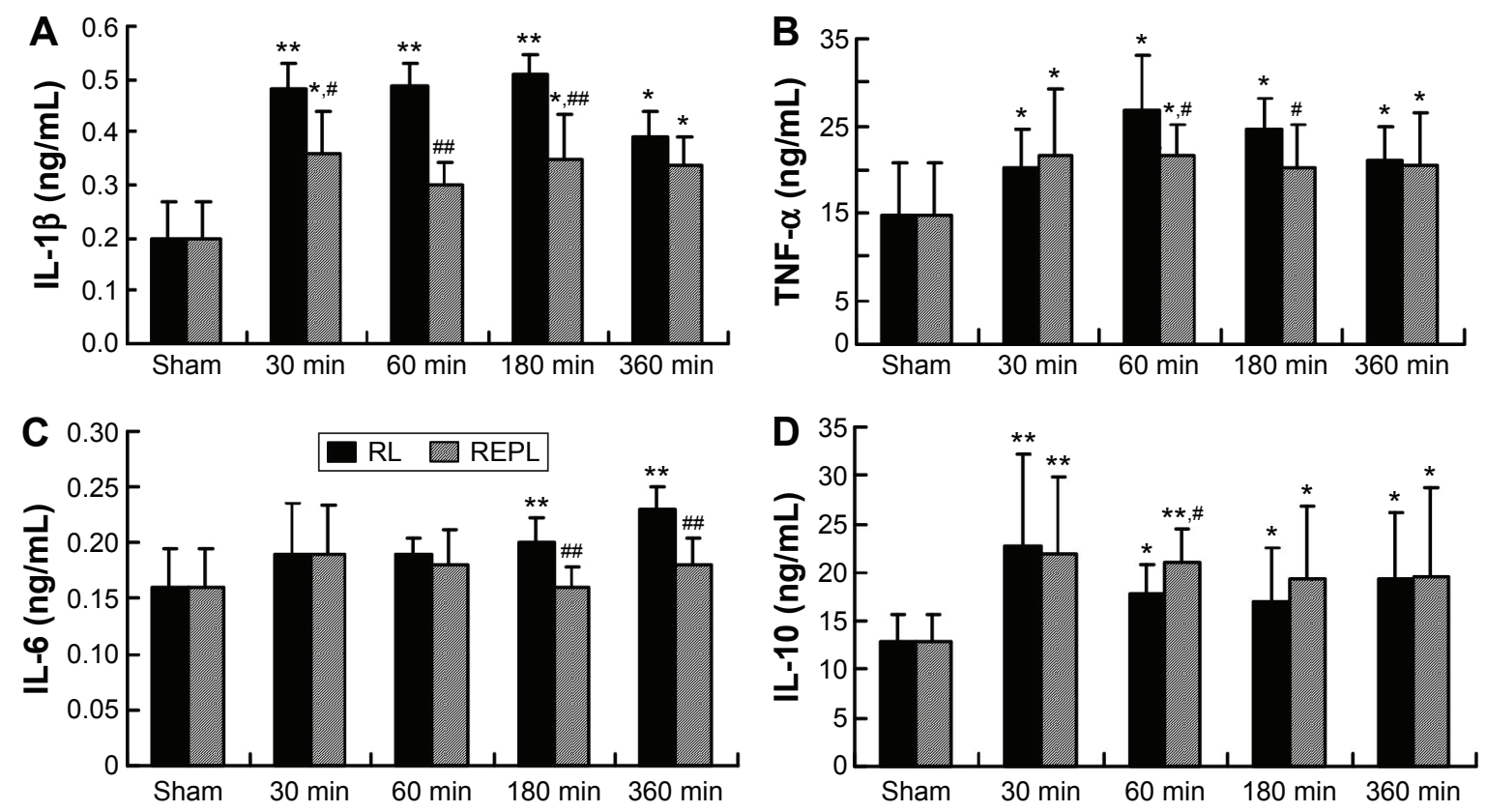

Figure 2 Effects of EP on the levels of inflammatory factors in the plasma of septic mice.

Notes: The plasma of each mouse was collected, and the $(\mathbf{A}) \mathrm{IL}-\mathrm{I} \beta,(\mathbf{B}) \mathrm{TNF},(\mathbf{C}) \mathrm{IL}-6$, and (D) IL-I0 levels were assayed using radioimmunoassay kits. The results are presented as the mean $\pm S D(n=10)$. $* P<0.05$ and ${ }^{* *} P<0.01$ versus the Sham group; ${ }^{*} P<0.05$ and ${ }^{\# \#} P<0.01$ versus the RL group.

Abbreviations: EP, ethyl pyruvate; IL, interleukin; TNF, tumor necrosis factor; SD, standard deviation; RL, Ringer's lactate solution; REPL, RL containing EP; min, minutes. 
A

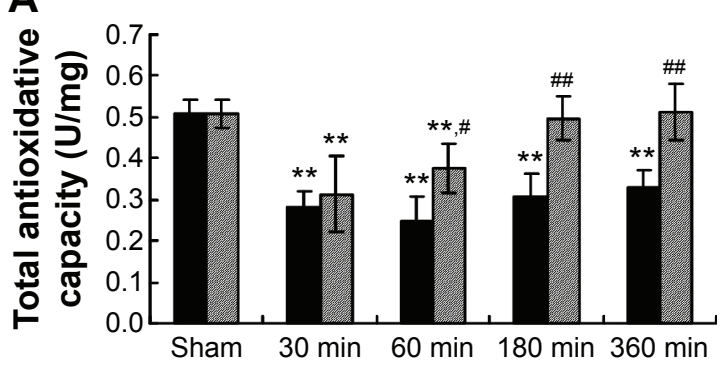

C

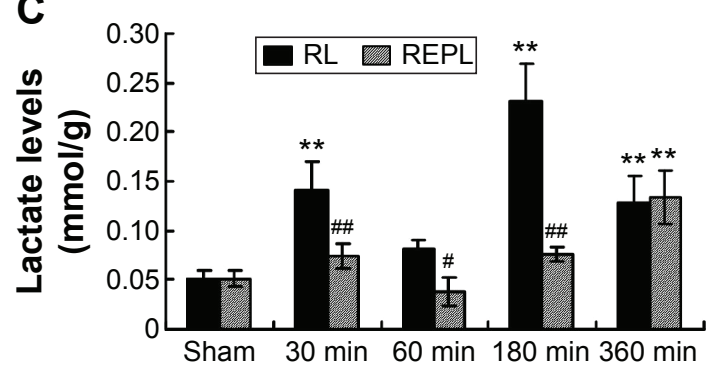

B

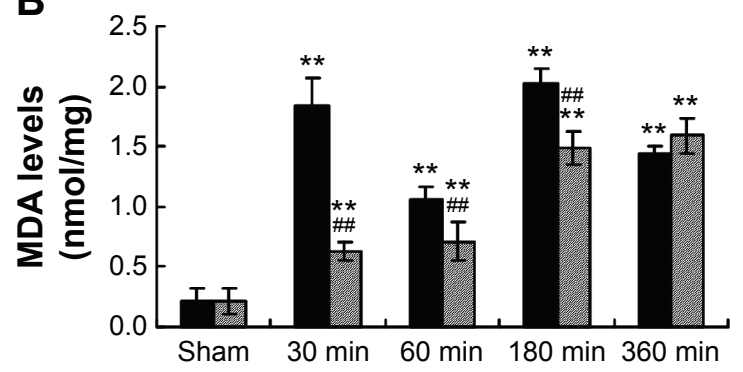

D

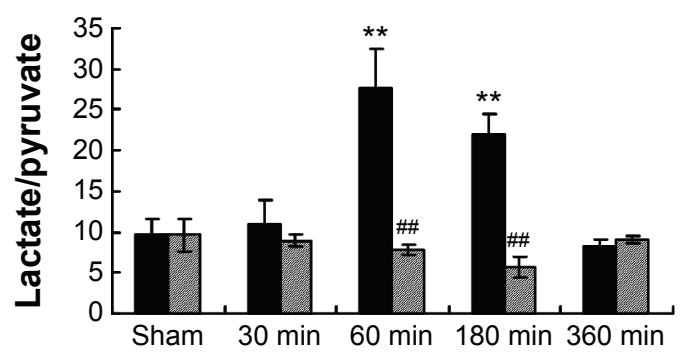

Figure 3 Effects of EP on oxidative damage and metabolic acidosis in the liver of septic mice.

Notes: The liver homogenates of each mouse were collected, and the (A) TAC, (B) MDA, (C) lactate, and (D) lactate/pyruvate levels were assayed using respective colorimetric assay kits. The results are presented as the mean $\pm S D(n=10)$. ${ }^{*} P<0.05$ and ${ }^{* * P}<0.01$ versus the Sham group; ${ }^{*} P<0.05$ and ${ }^{\#} P<0.01$ versus the $R L$ group. Abbreviations: EP, ethyl pyruvate; TAC, total antioxidative capacity; MDA, malondialdehyde; SD, standard deviation; RL, Ringer's lactate solution; REPL, RL containing EP; min, minutes.

\section{EP reduces depletion of the energy metabolism in the liver of septic mice}

Sepsis induces a high metabolic rate in an organism. To explore the novel protective effects of EP against sepsis, the concentration levels of ATP, AMP, and ADP were detected, and the TAN and EC levels were calculated by comparing the peak area of the sample peaks with calibration curves for the peak areas of each standard compound (Figure 4A). Our results demonstrated that, in a comparison with the Sham group, the ATP (Figure 4B) levels in the RL groups were significantly decreased from 30 minutes after CLP to 360 minutes, and the TAN (Figure 4C) and EC (Figure 4D) levels presented a pattern similar to that obtained for the ATP levels. The EP treatment significantly increased the ATP, TAN, and EC levels in the liver of septic mice compared with those of the RL groups.

\section{Discussion}

In this study, we demonstrated that EP is a potential antiinflammatory and antioxidative agent in CLP-induced septic mice, as was indicated by improvements in the survival rate as well as intestinal histological alterations, inhibition of pro-inflammatory cytokines, and ROS. In addition to its antiinflammatory and antioxidant effects, EP may function as an energy metabolism substrate to protect against sepsis.

Sepsis may cause hypotension and multiorgan dysfunction leading to septic shock and death. CLP-induced sepsis is a classical injury model. It initially induces the structural and functional derangements. Previous studies have demonstrated that EP administration markedly protects gut epithelial barrier from intestinal ischemic injury. ${ }^{4} \mathrm{We}$ observed that CLP induced time-dependent damage to the intestinal mucosa in mice which was indicated by massive inflammatory cell infiltration, epithelial necrosis, and lifting down of the sides of the villi. Although EP treatment could not reverse the damage to the intestinal mucosa, it could improve the serious injury, particularly in the advanced stages. After intestinal barrier damage, large amount of endotoxemia and ROS release into bloodstream that would induce systemic inflammatory reaction and multiorgan dysfunction. In clinic, acute septic shock syndrome occurs suddenly, and a patient dies within 24-48 hours mainly due to the sudden overproduction of proinflammatory cytokines and ROS., ${ }^{2,17}$ Proinflammatory cytokine and oxygenic free radical overproduction induces systemic inflammatory responses and metabolic disorders at as early as 6 hours after injury. ${ }^{18}$ In this study, we demonstrated that EP administration significantly inhibited the elevation of proinflammatory and oxygenic free radicals as early as 30 minutes after CLP. Previous studies have demonstrated that EP attenuates systemic inflammation after the induction of sepsis, and this is associated with significant reductions in the circulating levels of TNF- $\alpha$ and HMGB. ${ }^{19,20}$ In rats challenged with intravenous lipopolysaccharide to induce endotoxic shock, EP treatment resulted in longer survival, significantly decreased the circulating concentrations of nitric 
A

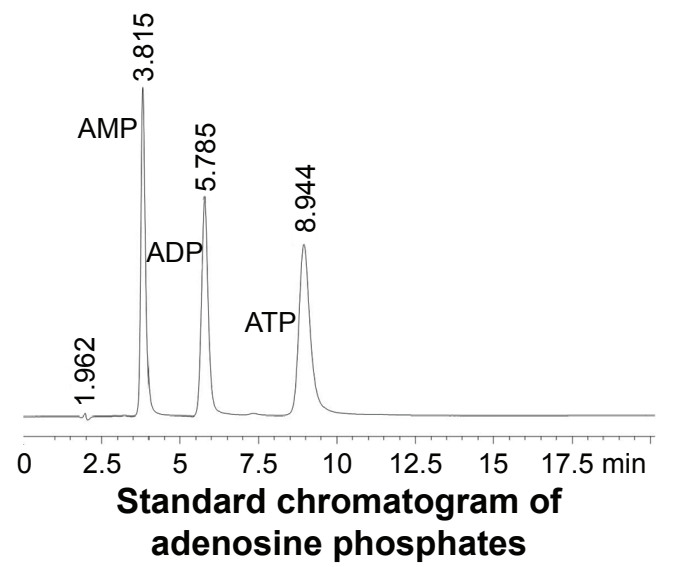

C

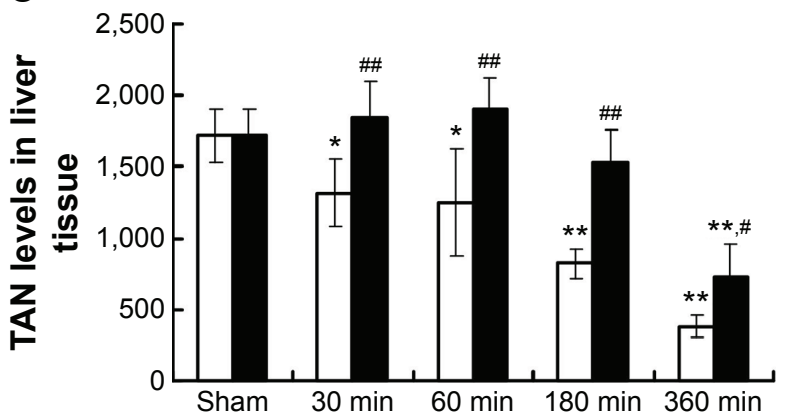

B

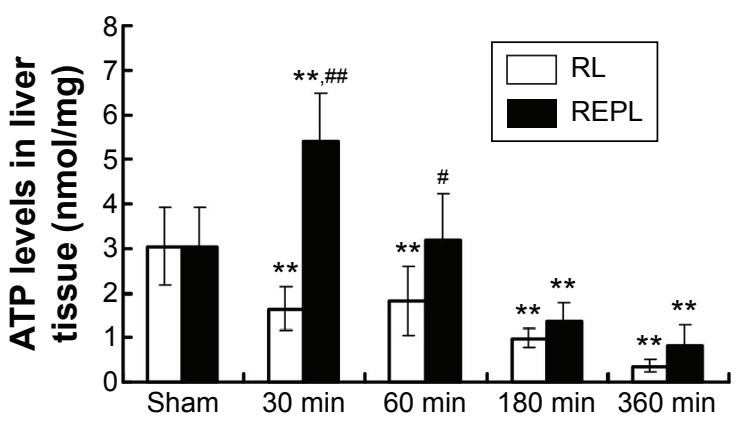

D

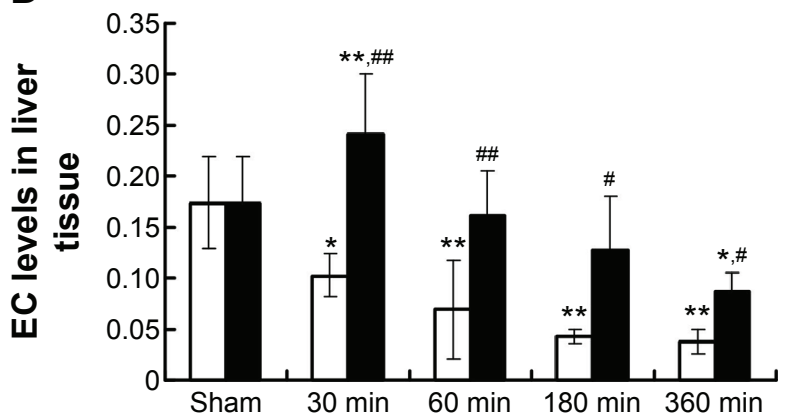

Figure 4 Effects of EP on the energy metabolism in the liver of septic mice.

Notes: The ATP, TAN, and EC levels in the livers were determined by HPLC. (A) Standard chromatogram of adenosine phosphates. Wavelength $=259$ nm. (B) ATP dissipation in the livers at each time point. (C) Change in the TAN levels in the livers. TAN $=$ ATP + AMP + ADP. (D) Change in the EC levels in the livers. EC $=($ ATP + I/2ADP)/TAN ( $n=10$ for each group). $* P<0.05$ and ${ }^{*} * P<0.01$ versus the Sham group; ${ }^{\#} P<0.05$ and ${ }^{\#} P<0.01$ versus the $R L$ group.

Abbreviations: EP, ethyl pyruvate; ATP, adenosine triphosphate; TAN, total adenylate; EC, energy charge; HPLC, high-performance liquid chromatography; AMP, adenosine monophosphate; ADP, adenosine diphosphate; RL, Ringer's lactate solution; REPL, RL containing EP; min, minutes.

oxide and IL-6, which are pro-inflammatory molecules, and increased the plasma levels of IL-10, an anti-inflammatory cytokine. ${ }^{21}$ Disruption of the energy metabolism is an important hallmark of sepsis because a restricted or high metabolic rate inhibits the synthesis of ATP molecules, which are required for the maintenance of ionic gradients and cell survival. ${ }^{8}$ Ozawa et al demonstrated that the energy metabolism of the liver after injury plays a key role in the maintenance of homeostasis. ${ }^{22}$ Liver functions are very complex, and the liver is considered the biochemical factory of the body. Many metabolic reactions, such as glycogen synthase, detoxification, and glucose regulation, are performed in the liver. The metabolic activities of organs are increased after injury, but the functions of the liver are retained, which could produce energy by the degradation of fatty acids and support the functions of other organs. ${ }^{23}$ Thus, the maintenance of the energy metabolism is more important in the liver than in other organs. In this study, we detected metabolic acidosis-associated lactate and lactate/pyruvate in the livers of septic mice. The levels of lactate and lactate/pyruvate in the livers were significantly increased after CLP compared with those in the
Sham group. The EP treatment reversed metabolic acidosis, as demonstrated by significantly reduced lactate and lactate/ pyruvate levels. After CLP, the increased oxygen and glucose consumption leads to oxidative phosphorylation disorders, resulting in energy reduction and even energy exhaustion, which increases anaerobic glycolysis and leads to lactic acid accumulation and subsequently to the induction of tissue acidosis and injury. ${ }^{24}$ The lactate/pyruvate ratio is an important parameter of the liver, and a ration $\geq 25$ indicates the onset of anaerobic metabolism..$^{25}$ ATP is the most important energy molecule in tissues and is needed for the maintenance of essential cellular functions. Many previous studies have demonstrated that the tissue ATP levels generally decrease in the early stage after injury. ${ }^{26,27}$ Our results demonstrated that the ATP levels in the liver were significantly decreased starting from 30 minutes after CLP to 360 minutes compared with the Sham group, and the TAN and EC levels present patterns that were similar to that found for the ATP levels. Shen et al demonstrated that EC is a parameter of cellular energy-rich phosphate binding, which indicates the energy state of the ATP-ADP-AMP system, and decreases in ATP 
and EC levels indicate energy overdepletion. ${ }^{28}$ Previous studies have demonstrated the ability of EP to enhance the ATP levels in a model of prolonged myocardial ischemia/ reperfusion injury. ${ }^{29}$ In this study, our results demonstrate that the EP treatment significantly increases the ATP, TAN, and EC levels in the livers of septic mice. These results suggest that in addition to its anti-inflammatory and antioxidant effects, EP may function as an energy metabolism substrate to protect against sepsis.

Our present study has some limitations. For example, we studied only one dosage amount of EP. Additionally, whether the impact of EP was direct or indirect was unclear. Moreover, we did not explore the mechanism by which EP regulated energy metabolism. These limitations should be further studied in the future. Although EP was shown to be safe, and many previous studies have demonstrated its beneficial effects on various injury models of animals, the application in clinic was limited. Most importantly, the pharmacological basis of EP remains to be elucidated. The poor solubility in pure water may be another reason for the paucity of clinical trials with EP, and its interactions with other elements in complex biological systems have not yet been characterized.

\section{Conclusion}

Although the detailed mechanisms are not clearly demonstrated, our results at least offer a novel explanation of how EP administration modulates energy metabolism in CLP-induced septic mice. EP can alleviate tissue acidosis and enhance the ATP levels, thereby repairing the organ energy deficit to protect against sepsis. In addition to its anti-inflammatory and antioxidant effects, EP is a potential multifunctional agent for critical diseases such as sepsis.

\section{Disclosure}

The authors report no conflicts of interest in this work.

\section{References}

1. Shapiro N, Howell MD, Bates DW, et al. The association of sepsis syndrome and organ dysfunction with mortality in emergency department patients with suspected infection. Ann Emerg Med. 2006; 48(5):583-590.

2. Kleinpell RM, Graves BT, Ackerman MH. Incidence, pathogenesis, and management of sepsis: an overview. AACN Adv Crit Care. 2006;17(4):385-393.

3. Olivencia-Yurvati AH, Blair JL, Baig M, Mallet RT. Pyruvate-enhanced cardioprotection during surgery with cardiopulmonary bypass. J Cardiothorac Vasc Anesth. 2003;17(6):715-720.

4. Sims CA, Wattanasirichaigoon S, Menconi MJ, Ajami AM, Fink MP. Ringer's ethyl pyruvate solution ameliorates ischemia/reperfusioninduced intestinal mucosa injury in rats. Crit Care Med. 2001;29(8): 1513-1518.
5. Tawadrous ZS, Delude RL, Fink MP. Resuscitation from hemorrhagic shock with Ringer's ethyl pyruvate solution improves survival and ameliorates intestinal mucosal hyperpermeability in rats. Shock. 2002;17(6): 473-477.

6. Guo J, Zhang J, Luo X, et al. Effects of ethyl pyruvate on cardiac function recovery and apoptosis reduction after global cold ischemia and reperfusion. Exp Ther Med. 2014;7(5):1197-1202.

7. Hu X, Cui B, Zhou X, et al. Ethyl pyruvate reduces myocardial ischemia and reperfusion injury by inhibiting high mobility group box 1 protein in rats. Mol Biol Rep. 2012;39(1):227-231.

8. Pravda J. Metabolic theory of septic shock. World J Crit Care Med. 2014;3(2):45-54.

9. Wang X, Qin W, Qiu X, et al. A novel role of exogenous carbon monoxide on protecting cardiac function and improving survival against sepsis via mitochondrial energetic metabolism pathway. Int J Biol Sci. 2014;10(7):777-788.

10. Crouser ED. Mitochondrial dysfunction in septic shock and multiple organ dysfunction syndrome. Mitochondrion. 2004;4(5-6):729-741.

11. Rittirsch D, Huber-Laang MS, Flierl MA, Ward PA. Immunodesign of experimental sepsis by cecal ligation and puncture. Nat Protoc. 2009; 4(1):31-36.

12. Uchiyama T, Delude RL, Fink MP. Dose-dependent effects of ethyl pyruvate in mice subjected to mesenteric ischemia and reperfusion. Intensive Care Med. 2003;29(11):2050-2058.

13. Chiu CJ, Mc Ardle AH, Brown R, Scott HJ, Gurd FN. Intestinal mucosal lesion in low-flow states. I. A morphological, hemodynamic, and metabolic reappraisal. Arch Surg. 1970;101(4):478-483.

14. Kammermeier H. Meaning of energetic parameters. Basic Res Cardiol. 1993;88(5):380-384.

15. Manfredi G, Yang L, Gajewski CD, Mattiazzi M. Measurements of ATP in mammalian cells. Methods. 2002;26(4):317-326.

16. Zur Nedden S, Eason R, Doney AS, Frenguelli BG. An ion-pair reversed-phase HPLC method for determination of fresh tissue adenine nucleotides avoiding freeze-thaw degradation of ATP. Anal Biochem. 2009;388(1):108-114.

17. Angus DC, van der Poll T. Severe sepsis and septic shock. $N$ Engl J Med. 2013;369(9):840-851.

18. Haupt W, Fritzsche H, Hohenberger W, Zirngibl H. Selective cytokine release induced by serum and separated plasma from septic patients. Eur J Surg. 1996;162(10):769-776.

19. Ulloa L, Ochani M, Yang H, et al. Ethyl pyruvate prevents lethality in mice with established lethal sepsis and systemic inflammation. Proc Natl Acad Sci U S A. 2002;99(19):12351-12356.

20. Fink MP. Ethyl pyruvate: a novel anti-inflammatory agent. J Intern Med. 2007;261(4):349-362.

21. Venkataraman R, Kellum JA, Song M, Fink MP. Resuscitation with Ringer's ethyl pyruvate solution prolongs survival and modulates plasma cytokine and nitrite/nitrate concentration in a rat model of lipopolysaccharide-induced shock. Shock. 2002;18(6):507-512.

22. Ozawa K, Fujimoto T, Nakatani T, et al. Changes in hepatic energy charge, blood ketone ratio, and indocyanine green clearance in relation to DNA synthesis after hepatectomy. Life Sci. 1982;31(7): 647-653.

23. Minemura M, Tajiri K, Shimizu Y. Liver involvement in systemic infection. World J Hepatol. 2014;6(9):632-642.

24. Waelgaard L, Dahl BM, Kvarstein G, Tonnessen T. Tissue gas tension and tissue metabolites for detection of organ hypoperfusion and ischemia. Acta Anaesthesiol Scand. 2012;56(2):200-209.

25. Burša F, Pleva L, Máca J, Sklienka P, Ševčík P. Tissue ischemia microdialysis assessments following severe traumatic haemorrhagic shock: lactate/pyruvate ratio as a new resuscitation end point. $B M C$ Anesthesiol. 2014;14:118-126.

26. Crawford RS, Albadawi H, Atkins MD, et al. Postischemic treatment with ethyl pyruvate prevents adenosine triphosphate depletion, ameliorates inflammation, and decreases thrombosis in a murine model of hind-limb ischemia and reperfusion. J Trauma. 2011;70(1): $103-110$. 
27. Sheth H, Hafez T, Glantzounis GK, et al. Glycine maintains mitochondrial activity and bile composition following warm liver ischemiareperfusion injury. J Gastroenterol Hepatol. 2011;26(1):194-200.

28. Shen LC, Fall L, Walton GM, Atkinson DE. Interaction between energy charge and metabolite modulation in the regulation of enzymes of amphibolic sequences. Phosphofructokinase and pyruvate dehydrogenase. Biochemistry. 1968;7(11):4041-4045.
29. Woo YJ, Taylor MD, Cohen JE, et al. Ethyl pyruvate preserves cardiac function and attenuates oxidative injury after prolonged myocardial ischemia. J Thorac Cardiovasc Surg. 2004;127(5):1262-1269.

\section{Publish your work in this journal}

Therapeutics and Clinical Risk Management is an international, peerreviewed journal of clinical therapeutics and risk management, focusing on concise rapid reporting of clinical studies in all therapeutic areas, outcomes, safety, and programs for the effective, safe, and sustained use of medicines. This journal is indexed on PubMed Central, CAS,
EMBase, Scopus and the Elsevier Bibliographic databases. The manuscript management system is completely online and includes a very quick and fair peer-review system, which is all easy to use. Visit http://www.dovepress.com/testimonials.php to read real quotes from published authors.

Submit your manuscript here: http://www.dovepress.com/therapeutics-and-clinical-risk-management-journal 